\title{
DYNAMICS OF QUANTUM SELECTED LEVELS OF ELECTRONIC EXCITED STATES OF SMALL MOLECULES
}

\author{
E. MARTÍNEZ, $\dagger$ M. R. LÓPEZ, $\dagger$ F. BASTERRECHEA \\ and P. PUYUELO $\ddagger$ \\ $\dagger$ Facultad de Químicas. Paseo de la Universidad, 4. Universidad \\ de Castilla-La Mancha. 13071 Ciudad Real. Spain \\ $\ddagger$ Universidad Pais Vasco. Apartado 644. Bilbao. Spain
}

\begin{abstract}
Dynamics studies of the electronic excited states of some small molecules have been carried out. Collision free lifetimes of different rotational and vibrational levels of the $\mathrm{A}$ and $\mathrm{B}$ states of ${ }^{130} \mathrm{Te}_{2}$ and ${ }^{80} \mathrm{Se}_{2}$ have been obtained. Predissociation studies of the $\mathrm{B}$ states of the $\mathrm{Br}_{2}$ and $\mathrm{Cl}_{2}$ molecules have been carried out. Results are discussed in terms of perturbation or predissociation effects.
\end{abstract}

KEY WORDS: Lifetime, fluorescence, selenium, tellurium, chlorine, bromine.

\section{INTRODUCTION}

Defined ro-vibrational $\left(v^{\prime}, \mathrm{J}^{\prime}\right)$ levels of some electronic excited states of small molecules can be populated by using narrow-band tunable dye lasers. Quantumresolved laser induced fluorescence (LIF) studies of certain excited states of some molecules, ${ }^{130} \mathrm{Te}_{2},{ }^{80} \mathrm{Se}_{2}, \mathrm{Br}_{2}, \mathrm{Cl}_{2}$, have been carried out in our laboratory. ${ }^{1-3}$ From direct observation of the undispersed fluorescence from these states radiative lifetimes have been obtained.

The dimers of sulphur, selenium and tellurium (group VI elements) that show broad spectral bands in absorption and emission are possible laser candidates for UV-to visible converter systems. However, few dynamics investigations have been made of the heavier diatomic molecules of this group. The photochemical and chemiluminiscent properties of the diatomic halogens $\mathrm{Br}_{2}$ and $\mathrm{Cl}_{2}$ are largely determined by the dynamics properties of their rovibrational excited states, i.e. rates for radiative transitions, spontaneous dissociation, collisional deactivation etc. The $\mathrm{B}$ states of $\mathrm{Br}_{2}$ and $\mathrm{Cl}_{2}$ are very familiar to photochemists, since the $\mathrm{B}-\mathrm{X}$ transitions of these molecules account for much of the well-known absorption spectra of $\mathrm{Br}_{2}$ and $\mathrm{Cl}_{2}$ in the visible regions.

\section{EXPERIMENTAL}

The experimental set-up has been described in previous works. ${ }^{1-5}$ Tellurium and selenium dimers were prepared by heating in a Pyrex or quartz cell into a home made oven, tellurium $(600-740 \mathrm{~K})$ and selenium $(\mathrm{T}>773 \mathrm{~K})$. Bromine and chlorine 
samples were prepared without further purification and pressure was controlled by a capacitance manometer. Dye lasers pumped by Nd - YAG laser or excimer lasers, delivering short pulses (15-20 ns) of 1-10 mJ, of narrow linewidth $0.15-0.08 \mathrm{~cm}^{-1}$, were used for excitation. Undispersed fluorescence from excited states was detected with a photomultiplier tube EMI $9816 \mathrm{~B}$, after filtering scattered light. Laser excitation spectra were obtained by recording fluorescence intensity through a boxcar integrator as a function of laser wavelength. A Tektronix 2430 A digital oscilloscope was used to acquire the fluorescence decays from the photomultiplier output. The signals were conveniently averaged and processed with a microcomputer to get the fluorescence lifetimes through standard statistical programs.

\section{RESULTS}

The $\mathrm{B}\left(\mathrm{O}_{u}^{+}\right)$and $\mathrm{A}\left(\mathrm{O}_{u}^{+}\right)$States of ${ }^{130} \mathrm{Te}_{2}$ and ${ }^{80} \mathrm{Se}_{2}$

Short and long-delay gated laser induced fluorescence (LIF) spectra were recorded by means of a boxcar integrator. In the spectral region studied $(465-496 \mathrm{~nm})$, the $\mathrm{A}-\mathrm{X}$ and $\mathrm{B}-\mathrm{X}$ band systems of $\mathrm{Te}_{2}$ are expected to be excited, the intensity of the $\mathrm{B}-\mathrm{X}$ transition being clearly stronger than that of the $\mathrm{A}-\mathrm{X}$ transition. On the other hand in the $503-520 \mathrm{~nm}$ region the A state emission is becoming dominant. The delayed gated intensity spectrum is clearly in favour of the long lived radiating state that is used to assign $\mathrm{A}-\mathrm{X}$ transition, where overlapped with the $\mathrm{B}-\mathrm{X}$ transition, in addition the data for the $\mathrm{A}, \mathrm{B}$ and $\mathrm{X}$ states of tellurium dimers were used to calculate transition frequencies and intensities for the $\mathrm{A}-\mathrm{X}$ and $\mathrm{B}-\mathrm{X}$ band systems.

For natural isotopic abundance, observed lifetimes of $\mathrm{Te}_{2}$ have been reported by E. Martínez et al.$^{1-2}$ A tentative value for the collision free lifetime of the B state of 80 $\pm 6 \mathrm{~ns}$ is proposed. On the other hand a radiative lifetime of $\tau=1080 \pm 60 \mathrm{~ns}$ has been proposed for emission of the low vibrational levels $v^{\prime}=5-10$ of the A state. As going up in the vibrational ladder, lifetimes become shorter, namely around $650 \mathrm{ns,}$ for emission from $v^{\prime}=10-14$ of the A state. After pumping the $v^{\prime}=14-18$ vibrational levels, no emission of the A state seems to be present or the fluorescence lifetimes for these levels of the A state are similar to those of the B state, i.e. $80 \mathrm{~ns}$. It would be compatible with the values presented by Cariou et al.$^{8}$ of $60-70 \mathrm{~ns}$ for emission from the $v^{\prime}=16,17$ vibrational levels of the A state. Similar studies have been made with the ${ }^{130} \mathrm{Te}_{2}$ enriched species and collision free lifetimes of the A state have been obtained ranging from $950 \mathrm{~ns}$ for $v^{\prime}=7$ to $760 \mathrm{~ns}$ for $v^{\prime}=13$. No rotational dependence of the lifetimes is observed, as shown in Table 1. Thus, while the low lying vibrational levels of the A state do not seem to be strongly perturbed, a substantial reduction of lifetimes is found for higher vibrational levels reaching values of $\tau<100 \mathrm{~ns}$ for $v^{\prime}=14-18$, indicating a serious perturbation. However the nature of such a perturbation should not be heterogeneous, since the perturbation mechanisms do not seem to be rotationally dependent. No variation is observed for the radiative lifetimes of the B state, with the rotational energy (see Table 1) or with the vibrational quantum number in the range $v^{\prime}=0-5$, obtaining an average value of $80 \pm 10 \mathrm{~ns}$. 
Table 1 Collision free lifetimes of selected rotational levels of the $\mathrm{A}\left(\mathrm{O}_{u}^{+}\right)$state vibrational level $v^{\prime}=8$, and of the $\mathrm{B}\left(\mathrm{O}_{u}^{+}\right)$state $v^{\prime}=4$ of the ${ }^{130} \mathrm{Te}_{2}$ molecule

\begin{tabular}{llll}
\hline \multicolumn{5}{c}{${ }^{130} \mathrm{Te}_{2}$} \\
\cline { 1 - 2 }$A\left(O_{u}^{+}\right)$state & & \multicolumn{3}{c}{$B\left(O_{u}^{+}\right)$state } \\
\cline { 1 - 2 }$v^{\prime}=8$ & $\tau_{O}(n s)$ & $v^{\prime}=4$ & $\sigma_{O}(n s)$ \\
\hline $\mathbf{J}^{\prime}=39$ & $900 \pm 10$ & $\mathbf{J}^{\prime}=43$ & $79 \pm 2$ \\
$\mathbf{J}^{\prime}=41$ & $939 \pm 13$ & $\mathbf{J}^{\prime}=45$ & $81 \pm 2$ \\
$\mathbf{J}^{\prime}=43$ & $941 \pm 19$ & $\mathbf{J}^{\prime}=47$ & $72 \pm 3$ \\
$\mathbf{J}^{\prime}=47$ & $897 \pm 18$ & $\mathbf{J}^{\prime}=61$ & $81 \pm 1$ \\
$\mathbf{J}^{\prime}=55$ & $866 \pm 13$ & $\mathbf{J}^{\prime}=65$ & $76 \pm 1$ \\
$\mathbf{J}^{\prime}=59$ & $891 \pm 9$ & & \\
$\mathbf{J}^{\prime}=67$ & $840 \pm 9$ & & \\
$\mathbf{J}^{\prime}=85$ & $854 \pm 20$ & & \\
$\mathbf{J}^{\prime}=87$ & $839 \pm 20$ & & \\
\hline
\end{tabular}

Laser excitation spectra of $\mathrm{Se}_{2}$ have been obtained, at $\mathrm{T}>773 \mathrm{~K}$ in an oven, by maintaining selenium liquid in a finger a $T=493 \mathrm{~K}$. A-X and $\mathrm{B}-\mathrm{X}$ band transitions have been assigned in a short wavelength interval around $388 \mathrm{~nm}$. Lifetimes of 80-90 ns were obtained for different rotational levels of the $v^{\prime}=2,3,4,5$, of the B state, finding no dependence with the rotational or vibrational quantum state. On the other hand, values of lifetimes around $450 \mathrm{~ns}$ were found for emission from rotational levels of $v^{\prime}=14$ of the A state.

\section{Predissociation of the $\mathrm{B}^{3} \Pi\left(\mathrm{O}_{u}^{+}\right)$States of $\mathrm{Br}_{2}$ and $\mathrm{Cl}_{2}$}

Laser excitation spectra of $\mathrm{Br}_{2}$ between 639 and $690 \mathrm{~nm}$ were recorded and several rotational transition for the emission from $v^{\prime}=2,3,4,6$ and 7 vibrational levels were assigned. Collision free lifetimes for emission from the stable level $v^{\prime}=2$ of around $\tau=10.4 \mu \mathrm{s}$ were obtained. No dependence from the rotational energy was found. However the observed lifetimes at low pressures for the levels $v^{\prime}=3,4,6$ and 7 were really shorter, showing a dependence that for $v^{\prime}=3$ and 4 can be put clearly in the form

$$
\frac{1}{\tau_{\mathrm{O}}}=\frac{1}{\tau_{\mathrm{R}}}+\mathrm{K}_{v}{ }^{\prime} \mathbf{J}^{\prime}\left(\mathbf{J}^{\prime}+1\right)
$$

where $\tau_{\mathrm{R}}$ is the purely radiative lifetime, and $\tau_{\mathrm{O}}$ the collision free lifetime. Values of $\mathrm{K}_{3}=(0.034 \pm 0.004) \times 10^{3} \mathrm{~s}^{-1}$ and $\mathrm{K}_{4}=(4.38+0.67) \times 10^{3} \mathrm{~s}^{-1}$ for $v^{\prime}=3$ and $v^{\prime}=4$ respectively were obtained. A more complex dependence in $v^{\prime}=6$ and $v^{\prime}=7$ was observed.

The experimental results can be explained in terms of the dependence of the predissociation rate in the form:

$$
\mathrm{A}_{n r}=\mathrm{K}_{v^{\prime}} \mathbf{J}^{\prime}\left(\mathbf{J}^{\prime}+1\right)=\mathrm{K}^{\prime} \mathrm{P}_{v} \mathbf{J}^{\prime}\left(\mathbf{J}^{\prime}+1\right)
$$

with $\mathrm{K}^{\prime}=\frac{\hbar^{2}}{4 \mu^{2} c}|\mathrm{We}(\overline{\mathrm{R}})|^{2}$ and $\mathrm{P}_{v^{\prime}}=\left|<\mathrm{X}_{c}\right| \mathrm{R}^{-2}\left|\mathrm{X}_{b}>\right|^{2}$ 


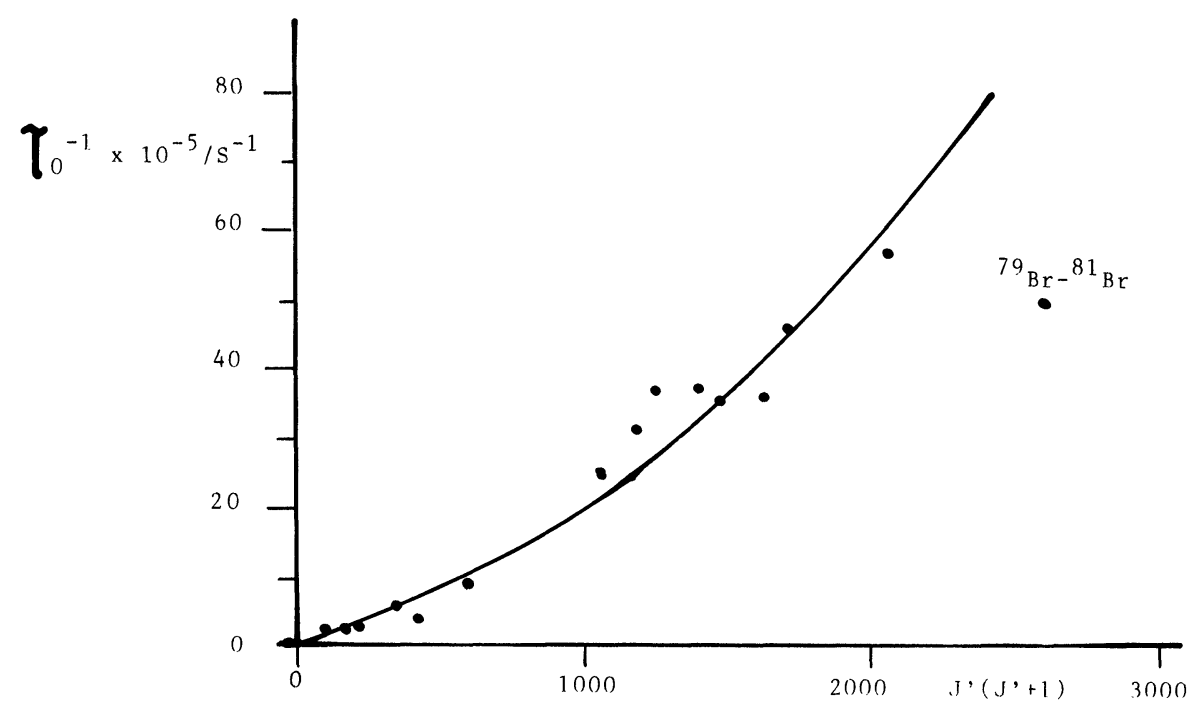

Figure 1 Inverse collision free lifetimes $\tau_{\mathrm{O}}{ }^{-1}$ versus $\mathrm{J}^{\prime}\left(\mathrm{J}^{\prime}+1\right)$ for the $v^{\prime}=6$ level of ${ }^{79} \mathrm{Br}-{ }^{81} \mathrm{Br} \mathrm{B}$ state. Solid line for calculated values.

where $\mathrm{R}$ is the internuclear distance, $\mathrm{X}_{c}$ and $\mathrm{X}_{b}$ are the vibrational wave functions of the continuum and bound states respectively, and $\mathrm{We}(\overline{\mathrm{R}})$ is a dimensionless quantity which involves only electronic operators and coordinates. $\mathrm{R}$ being the transition R-centroid. Calculations of $P_{V^{\prime}}$ values were made between the $B$ and ${ }^{1} \Pi$ states of bromine. The obtained experimental data, especially for $v^{\prime}=6$ and 7 , should be useful in order to check the validity of the repulsive curve ${ }^{1} \Pi$, as the $\mathrm{P}_{v^{\circ} \mathrm{J}^{\circ}}$ behaviour is very sensitive to small changes in the potential curve. See Figure 1. A slightly modified repulsive curve $\Pi$, with a crossing point at $E_{x}=610 \mathrm{~cm}^{-1}$ and $\mathrm{R}_{x}=2.954 \mathrm{~A}$ is proposed.

Table 2 Experimental predissociation rates $\mathrm{K}_{v^{\prime}}$ for different vibrational levels of B state

\begin{tabular}{lll}
\hline$K v^{\prime} 10^{5} s^{-1}$ & Our results & Clyne et al. $^{9}$ \\
\hline 13 & - & $7.7 \pm 0.4$ \\
14 & $7.78 \pm 0.71$ & $5.9 \pm 0.4$ \\
15 & $6.12 \pm 0.50$ & $5.3 \pm 0.4$ \\
16 & $5.69 \pm 0.15$ & $4.1 \pm 0.5$ \\
17 & $4.00 \pm 0.24$ & - \\
18 & $3.77 \pm 0.38$ & $3.8 \pm 0.5$ \\
19 & $2.24 \pm 0.12$ & $4.2 \pm 0.7$ \\
20 & - & - \\
21 & $2.34 \pm 0.37$ & $2.9 \pm 0.5$ \\
22 & - & $2.4 \pm 0.4$ \\
23 & - & $1.6 \pm 0.5$ \\
24 & - & $1.2 \pm 0.3$ \\
25 & - & $1.1 \pm 0.3$ \\
\hline
\end{tabular}


Chlorine $\mathrm{B}{ }^{3} \Pi\left(\mathrm{O}_{u}^{+}\right)$state lifetimes of specific $\left(v^{\prime}, \mathrm{J}^{\prime}\right)$ levels have been measured for several predissociated levels $\left(v^{\prime}=14-19,21\right)$. Experimental data fit quite well with Eq. (1) and $\mathrm{K}_{v^{\circ}}$ values are obtained as shown in Table 2. By using these values, trial and error Franck-Condon calculations have been performed to check the nature and position of the predissociating state. A best value of $\mathrm{R}_{x}=2.48 \pm 0.01 \AA$ for the $\mathrm{A}$ ${ }^{3} \Pi\left(1_{u}\right)$ state responsible of the B state predissociation is given.

\section{References}

1. E. Martínez, P. Puyuelo, F. J. Basterrechea and M. T. Martínez, Chem. Phys. Lett. 156, 564 (1989).

2. E. Martínez, F. J. Basterrechea, P. Puyuelo and F. Castaño, J. Phys. B. 23, 77 (1990).

3. E. Martínez, F. J. Basterrechea, J. Albaladejo, F. Castaño and P. Puyuelo, J. Phys. B. 24, 2765 (1991).

4. F. Castaño, E. Martínez and M. T. Martínez, Chem. Phys. Lett. 128, 137 (1986).

5. E. Martínez, M. T. Martínez and F. Castaño, J. Mol. Spectros, 128, 554 (1988).

6. J. Verges, C. Effantin, O. Babaky, J. d'Incan, S. J. Prosser and R. F. Barrow, Physica Scripta 25, 338 (1982).

7. A. Pardo, J. M. L. Poyato, M. J. Guijarro, J. J. Camacho and F. I. Fernández Alonso. An. Química 81A, 219 (1985).

8. J. Cariou and P. Luc, Atlas du Spectre d'absorption de la molecule de Tellure (Orsay: Laboratoire Aimé Cotton) (1980).

9. M. A. A. Clyne and E. Martínez. J. Chem. Soc. Faraday Trans. II 76, 1561 (1980). 